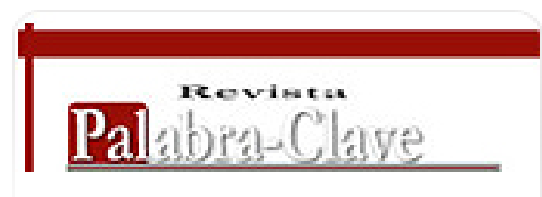

Palabra Clave

ISSN: 0122-8285

palabra.clave@unisabana.edu.co

Universidad de La Sabana

Colombia

Islas-Carmona, Jose Octavio

El prosumidor. El actor comunicativo de la sociedad de la ubicuidad

Palabra Clave, vol. 11, núm. 1, junio, 2008, pp. 29-39

Universidad de La Sabana

Bogotá, Colombia

Disponible en: http://www.redalyc.org/articulo.oa?id=64911103

- Cómo citar el artículo

Número completo

- Más información del artículo

- Página de la revista en redalyc.org

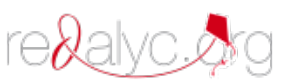

Sistema de Información Científica

Red de Revistas Científicas de América Latina, el Caribe, España y Portugal

Proyecto académico sin fines de lucro, desarrollado bajo la iniciativa de acceso abierto 


\title{
El prosumidor. El actor comunicativo de la sociedad de la ubicuidad
}

\section{The Prosumer: The Communicative Agent of the Ubiquitous Society}

\author{
Jose Octavio Islas-Carmona ${ }^{1}$
}

\author{
A Amaia Arribas. \\ Siempre indispensable
}

\section{Resumen}

En el imaginario de la sociedad de la ubicuidad, en el cual las comunicaciones digitales inciden de forma categórica en el desarrollo y la evolución de los nuevos ambientes comunicativos, las figuras de "emisor" y "receptor", así como los modelos que ayer permitían explicar el proceso comunicativo, hoy exhiben evidentes limitaciones en sus capacidades explicativas. La realidad los ha desbordado.

En el desarrollo histórico de los medios de comunicación es posible advertir cómo éstos resienten determinadas remediaciones. Desde la perspectiva de la ecología de medios, Internet -el medio de comunicación inteligente-, admite ser comprendido como lógica extensión del telégrafo. El telégrafo representó la primera exteriorización de nuestro sistema nervioso.

El desarrollo de las comunicaciones digitales móviles nos desplaza hacia un nuevo ambiente mediático: la "sociedad de la ubicuidad". De acuerdo con Neil Postman, detrás de toda tecnología subyace una filosofía, y los principios comunicativos de la "sociedad de la ubicuidad": comunicación para todos, en cualquier momento, en cualquier lugar, desplazan consigo la necesidad de producir los dispositivos de comunicaciones digitales móviles idóneos para responder a las exigencias del ambiente comunicativo que supone dicha sociedad.

El desarrollo de la web 2.0 admite ser considerado como un auténtico parteaguas histórico en la evolución de Internet; éste impuso importantes cambios tanto en el comportamiento como en los hábitos de consumo cultural de los cibernautas. El cibernauta accedió a la condición de prosumidor. Debemos reconocer a los prosumidores como los actores comunicativos de la sociedad de la ubicuidad. El papel de los prosumidores resultará definitivo en las siguientes remediaciones que experimentará Internet, medio que definitivamente admite ser comprendido como lógica extensión de la inteligencia humana.

Palabras clave: Internet, medios de comunicación, impacto de la comunicación, cambio tecnológico, proceso de comunicación. (Fuente: Tesauro de la UNESCO).

1 Doctor en Ciencias Sociales. Director de la Asociación Latinoamericana de Investigadores de la Comunicación (Alaic). Maestro en Comunicación y Desarrollo, Maestro en Administración y Tecnologías de Información, Director de Proyecto Internet-Cátedra de Comunicaciones Estratégicas y Cibercultura, Tecnológico de Monterrey, Campus Estado de México. octavio.islas@itesm.mx
Abstract

In the imaginary of the ubiquitous society, where digital communications exert a categorical influence on the development and evolution of new communication environments, the transmitter and the receiver, as well as the models once used to explain the communication process, now exhibit obvious limitations in their capacity to explain. The real world and existing circumstances have gone beyond them.

In the history of the development of the mass media, one can see a resistance to certain "remediations". From the standpoint of media ecology, the Internet - the intelligent medium of communication - can be understood as a logical extension of the telegraph, which was the first exteriorization of our nervous system.

The development of mobile digital communications has moved us into a new media environment: the ubiquitous society. According to Neil Postman, there is an underlying philosophy behind all technology, and the communicative principles of the ubiquitous society: communication for all, at any time and place, involve the need to produce mobile digital communication devices to satisfy the demands of the communication environment inherent in the ubiquitous society.

The development of Web 2 can be regarded as a genuine parting of the waters in the history of the evolution of the Internet. It introduced important changes in both the behavior and the cultural consumption habits of cybernauts. The cybernaut is a prosumer, and prosumers must be recognized as the communication agents of the ubiquitous society. They will play a decisive role in subsequent remediations of the Internet, a medium that definitely can be understood as a logical extension of human intelligence.

Key words: Internet, media, impact of communication, technological change, communication process.
Recibido: $24 / 04 / 2008$

Aprobado: 15/05/2008 


\section{El concepto remediación desde la perspectiva teórica de la ecología de medios}

Entre las distintas escuelas que en las ciencias de la comunicación se han ocupado de analizar de manera integral el proceso de la comunicación, la ecología de medios o "media ecology" se distingue por conceder particular énfasis al estudio de las tecnologías y los ambientes comunicativos, pues, como atinadamente afirmó Marshall McLuhan en el libro Comprender los medios de comunicación. Las extensiones del ser humano -cuya primera edición fue publicada en 1964-, en última instancia los medios de comunicación admiten ser comprendidos como tecnologías, y las tecnologías efectivamente pueden ser pensadas como prolongaciones del hombre. McLuhan, por ejemplo, afirmó que la televisión -el gigante tímido-, podía ser entendida como una extensión del sentido del tacto, considerando que la televisión implica una mayor interacción entre todos los sentidos (McLuhan, 1996, p. 338).

La ecología de medios, conocida también como "Escuela de Toronto", "Escuela de Nueva York", e incluso como "Escuela de San Luis", parte del pensamiento de Marshall McLuhan, para enriquecerse significativamente con las contribuciones de reconocidos académicos e intelectuales como Neil Postman, Joshua Meyrowitz, Paul Levinson, James W. Carey, Jay David Bolter y Lance Strate, entre otros. De acuerdo con Neil Postman, el objeto de estudio de la ecología de medios es el siguiente:

La ecología de los medios analiza como los medios de comunicación afectan la opinión humana, la comprensión, la sensación, y el valor;

La palabra "ecología" implica el estudio de los ambientes y sus interrelaciones: contenido, estructura, e impacto social. y cómo nuestra interacción con los medios facilita o impide nuestras posibilidades de supervivencia. La palabra ecología implica el estudio de ambientes: su estructura, contenido e impacto en la gente ${ }^{2}$.

Fernando Gutiérrez, director del Departamento de Comunicación y Arte Digital del Tecnológico de Monterrey, Campus Estado de México, y miembro del consejo directivo de la Media Ecology Association (MEA), define a la ecología de medios como una metadisciplina que se encarga del estudio de un complejo conjunto de relaciones o interrelaciones entre los símbolos, los medios y la cultura. La palabra "ecología" implica el estudio de los ambientes y sus interrelaciones: contenido, estructura, e impacto social. Un ambiente mediático es aquel que deriva de las interrelaciones entre el hombre y las distintas tecnologías de la comunicación, como libros, radio, televisión, Internet. La "ecología mediática" se refiere al estudio de las técnicas, los modos de información y los códigos de comunicación, como parte principal de un ambiente interrelacionado que proyecta diferentes efectos en un contexto determinado.

En el desarrollo de todo medio de comunicación es posible percibir cómo progresivamente el medio es transformado con el propósito, no siempre consciente o deliberado, de hacerlo más parecido al hombre. En la ecología de medios ese proceso es conocido como "remediación". El proceso de remediación de los medios de comunicación es el resultado de la adecuación dialéctica que permite transformarlos hasta convertirlos en lógicas extensiones de nuestras facultades, órganos o

2 En el sitio web de la Media Ecology Association (MEA) es posible consultar la definición de Neil Postman. Véase: http://www.media-ecology.org/media ecology [Fecha de consulta, 21 de abril de 2008]. Además, en el sitio web de la Media Ecology Association es posible consultar una interesante relación de los textos considerados como seminales. Véase: http://www.media-ecology.org/media_ecology/readinglist.html [Fecha de consulta: 21 de abril de 2008]. 
El proceso de remediación de los medios de comunicación es el resultado de la adecuación dialéctica que permite transformarlos hasta convertirlos en lógicas extensiones de nuestras facultades, órganos o sentidos.

sentidos. El concepto "remediación”, sin embargo, admite por lo menos dos posibles interpretaciones en ecología de medios.

En el libro The Soft Age, Paul Levinson (1997) empleó el término "medio remedial" para describir cómo nuestras sociedades utilizan los medios de comunicación para reformar o mejorar a otros medios. De acuerdo con Levinson, en un esfuerzo del cual no siempre somos conscientes, intentamos hacer que los medios se parezcan más al hombre. En un reciente libro: Cellphone. The story of the world's most mobile medium and how it has transformed everything, Levinson afirmó que los medios de comunicación aún compiten -y de forma más intensa- por la atención de las personas (Levinson, 2004, p. 12).

En términos darwinianos -sugiere Levinson-, la selección del ambiente mediático queda en manos de las personas, quienes contribuyen con su preferencia a la evolución de determinados medios. Constantemente se decide entre ir al cine o quedarse en casa a ver televisión, leer un libro o ver un video, hablar por celular o enviar un correo electrónico. Los medios no evolucionan por una selección natural sino por una elección humana. El medio que mejor evoluciona es aquel que se ajusta más a las necesidades del hombre.

Jay Bolter y Richard Grusin emplean el concepto "remediación" para describir las relaciones formales de interdependencia cultural que existen entre dos o varios medios, argumentando que en las primeras líneas del libro Comprender a los medios de comunicación. Las extensiones del ser humano, Marshall McLuhan afirmó que el contenido de todo medio es otro medio. Para Bolter y Grusin, Internet es un medio remediador, pues asimila a los medios que le antecedieron. Con base en tal razonamiento es posible afirmar que el contenido de Internet son los medios de comunicación que le precedieron.

De acuerdo con algunos destacados teóricos de la ecología de medios, Internet representa una avanzada remediación del telégrafo ${ }^{3}$, el cual fue designado por McLuhan como "la hormona social". Con el telégrafo, el hombre por primera vez logró extender su sistema nervioso central fuera de sí. Según McLuhan (1996, p. 260), el telégrafo, además, observó un rol definitivo en la transición de la edad mecánica a la edad eléctrica, pues:

revolucionó por completo los métodos de obtención y presentación de las noticias (...) Así, en 1844, año en el que se jugaba al ajedrez y a la lotería con el primer telégrafo estadounidense, Søren Kierkergaard publicó El concepto de la angustia. Había empezado la edad de la ansiedad. Con el telégrafo, el hombre había iniciado aquella extensión o exteriorización del sistema nervioso central que ahora se acerca a la exten-

Los medios no evolucionan por una selección natural sino por una elección humana. El medio que mejor evoluciona es aquel que se ajusta más a las necesidades del hombre.

3 El desarrollo del telégrafo en principio estuvo subordinado al periódico y al ferrocarril. En 1844 Samuel Morse abrió una línea telegráfica entre Washington y Baltimore. En 1858 ya se había tendido el primer cable que cruzaba el Atlántico, y tres años después, en 1861, los hilos telegráficos se extendían por toda la Unión Americana. El telégrafo revolucionó los sistemas de información y comunicaciones disponibles en su momento histórico. 
sión de la conciencia mediante la retransmisión por satélite. Colocar los nervios fuera del sistema nervioso y los órganos físicos dentro de éste es una situación -no un concepto- de angustia.

En cuanto al impacto cultural de los medios y las tecnologías de información, en general, en las primeras líneas del libro The medium is the Massage. An inventory of effects, Marshall McLuhan y Quentin Fiore $(1967$, p. 8) afirman:

El medio, o el proceso, de nuestro tiempo -la tecnología eléctrica- está alterando las pautas de reestructuración y de la interdependencia social y todos los aspectos de nuestra vida personal. Se nos obliga a reconsiderar y reevaluar prácticamente cada pensamiento, cada acción y cada institución. Todo está cambiando, usted, su familia, su barrio, su educación, su trabajo, su gobierno, su relación con los otros. Y están cambiando dramáticamente.

En años recientes, la evolución de Internet ha impuesto profundas transformaciones en la ecología cultural de las sociedades contemporáneas, modificando, incluso, la mayoría de conceptos que ayer nos permitían comprender la dinámica del proceso comunicativo. Quizá aún no seamos conscientes de cuán profundos son los cambios que han introducido Internet y las comunicaciones digitales móviles, en general, sobre las sociedades contemporáneas. Más importante aún, las transformaciones son irreversibles.

En años recientes, la evolución de Internet ha impuesto profundas transformaciones en la ecología cultural de las sociedades contemporáneas, modificando, incluso, la mayoría de conceptos que ayer nos permitían comprender la dinámica del proceso comunicativo.

\section{La sociedad de la ubicuidad: el nuevo ambiente comunicativo al cual nos conducirán las comunicaciones digitales móviles}

Una de las mejores explicaciones sobre la fenomenología de los cambios tecnológicos en las sociedades -tema medular en la ecología de medios - , procede de Neil Postman, uno de los pilares teóricos de la media ecology.

El 27 de marzo de 1998, Neil Postman, entonces decano del Departamento de Cultura y Comunicación de la Universidad de Nueva York, Estados Unidos, dictó, en Denver, Colorado, una de las conferencias magistrales del Congreso Internacional sobre Nuevas Tecnologías y Persona Humana: Comunicando la fe en el Nuevo Milenio, o NewTech'98.

El título de la conferencia dictada por Neil Postman fue "Five Things We Need to Know About Technological Change" (Cinco cosas que necesitamos conocer acerca del cambio tecnológico $)^{4}$. Las cinco tesis que enunció Postman en la referida conferencia son:

1. La cultura siempre paga el precio de la tecnología.

2. Siempre hay ganadores y perdedores en el cambio tecnológico.

3. Toda la tecnología tiene una filosofía.

4. El cambio tecnológico no es aditivo; es ecológico.

5. Los medios de comunicación tienden a convertirse en míticos.

Deseo centrar mi atención en la tercera tesis de Neil Postman, la cual nos permitirá comprender

4 Véase http://itrs.scu.edu/tshanks/pages/Comm12/12Postman.htm [Fecha de consulta: 20 de abril de 2008]. 
los fundamentos filosóficos que hacen posible sustentar, en Japón, el imaginario de la "sociedad de la ubicuidad".

Del 5 al 9 de octubre de 2004 se desarrollaron las actividades del Ceatec 2004, en Makuhari Messe, Japón. El Ceatec es la exhibición anual más importante en Asia de las industrias de electrónica avanzada y telecomunicaciones. Las principales marcas presentan nuevos productos que en breve introducirán al mercado. El tema central del Ceatec 2004 fue: "Sociedad digital ubicua enriquecida, acelerando la siguiente etapa"5.

En la referida edición del Ceatec 2004, Kunio Nakamura, presidente de Matsushita Electric Industrial Co. -corporativo del cual forma parte Panasonic-, dictó la conferencia magistral que inauguró las actividades de la referida feria tecnológica. El título del discurso de Nakamura fue el siguiente: "Creando la sociedad de la ubicuidad en Japón, una nación creada en la tecnología"; los japoneses se propusieron acceder a ella en el año 2010. El término "sociedad de la ubicuidad", afirma Nakamura, designa a una sociedad en la que cualquier persona puede disfrutar, en cualquier momento y en cualquier lugar, de una amplia gama de servicios de información a través de diversos dispositivos terminales y redes de banda ancha. La importancia de las comunicaciones digitales móviles se encuentra implícita en el lema de la sociedad de la ubicuidad: "anyone, anywhere, anytime" (cualquier persona, en cualquier lugar, en todo momento).

De acuerdo con Nakamura, tres factores resultan de capital importancia en el desarrollo de la sociedad de la ubicuidad: una sólida infraestructura de redes, eficientes dispositivos terminales, y servicios de contenido. La banda ancha admite ser considerada como la columna vertebral de la

5 Véase http://www.ceatec.com/es/2004/exhibitors/f-regulation.html. [Fecha de consulta: 20 de abril de 2008]. sociedad de la ubicuidad, y Japón es uno de los países que mayor cantidad de recursos ha destinado al desarrollo de una sólida infraestructura de redes de banda ancha. En 2001 el gobierno japonés puso en marcha la iniciativa "e-Japan Strategy". En la primera etapa de la referida iniciativa, el gobierno y la empresa privada destinaron los recursos necesarios para establecer una sólida infraestructura de servicios de banda ancha.

En la segunda etapa de "e-Japan Strategy", el gobierno decidió impulsar programas de alfabetización mediática para extender el uso de avanzadas tecnologías de información. Además, mediante el programa "e-Japan", los japoneses se han propuesto elevar las capacidades de las redes ubicuas, anticipándose así a la próxima generación de tecnologías de información. De acuerdo con Nakamura, según lo establecido en los planes en materia de telecomunicaciones, en 2010 las líneas fijas estarán en posibilidades de transmitir datos 10 veces más rápido que el ADSL. Además, las transmisiones inalámbricas serán 50 veces más rápidas que W-CDMA.

El impacto de e-Japón ha transformado la administración pública y la educación. Gracias a la incorporación de avanzados dispositivos digitales, el aparato administrativo-burocrático ha elevado significativamente su eficiencia. Un gran número de ciudadanos hoy realiza la mayoría de sus trámites gubernamentales en línea, sin necesidad alguna de desplazarse a las instituciones públicas.

\section{La banda ancha admite ser considerada como la columna vertebral de la sociedad de la ubicuidad, y Japón es uno de los países que mayor cantidad de recursos ha destinado al desarrollo de una sólida infraestructura de redes de banda ancha.}


En la educación, las avanzadas tecnologías de información han favorecido el desarrollo de un nuevo ambiente de aprendizaje: la educación móvil. La educación móvil supone el desarrollo de innovadores recursos de aprendizaje on demand, los cuales representan una lógica extensión de la sociedad de la ubicuidad.

Toda persona puede acceder a los recursos de aprendizaje disponibles en la red, a cualquier hora y en cualquier lugar. La educación móvil -en la cual dispositivos como el iPod observan un rol estelar-, impondrá profundos cambios tanto en la educación como en las instituciones educativas.

Japón además se ha propuesto acelerar el 1lamado "apagón analógico". La migración de las emisiones de televisión a la tecnología digital prácticamente se ha consumado. La radiodifusión digital terrestre se ha extendido a un mayor número de ciudades. Mientras las empresas de telefonía incrementan gigas a la capacidad de sus anchos de banda, en Japón se registra una notable expansión de la radiodifusión digital terrestre. La convergencia digital de ambas industrias, señala Nakamura, propiciará el desarrollo de un nuevo ambiente comunicativo, con una gran variedad de servicios.

Las transmisiones de televisión móvil por medio de dispositivos móviles -como los propios de la telefonía digital móvil- ${ }^{6}$, a través de FTTH, y de

6 En los años recientes el teléfono móvil ha registrado importantes transformaciones hasta convertirse en indispensable y multifuncional dispositivo de comunicaciones. En el imaginario de la sociedad de la ubicuidad, el rol del avanzado dispositivo admite ser considerado como fundamental. El teléfono móvil ha trascendido la condición de "terminal tonta", y hoy permite tener acceso a Internet; recibir y contestar correos electrónicos; asegurar el acceso a servicios de televisión móvil, mapas y avanzados servicios de localización; ser utilizado como cámara fotográfica, grabadora y avanzada remediación del popular walkman; asumir las funciones propias del mando a distancia, e incluso desempeñarse como útil dispositivo de almacenamiento de información digital. De las nuevas generaciones de inteligentes dispositivos de comunicación móvil seguramente derivarán nuevos usos y aplicaciones, como cartera digital para otras modalidades de banda ancha, se han vuelto muy populares en Japón. El éxito que alcanzó la serie Winter Sonata - una producción representativa del Broadband Drama Zoku (Broadband Drama Crowd)-, la cual originalmente fue transmitida a dispositivos móviles, estimuló en Corea del Sur el desarrollo de una atractiva industria de casas productoras especializadas en contenidos exclusivos para la televisión móvil nipona.

La mayoría de los contenidos destinados a la televisión móvil son servicios on demand. Ello significa que cualquier persona, en cualquier momento y en cualquier puede ver a través de dispositivos móviles las producciones o series que sean de su interés. No pocos medios convencionales han resultado incapaces de comprender la importancia del concepto on demand. En 2004 la cadena de televisión NHK ofrecía más de 400.000 programas en servicio on demand.

A pesar de los notables adelantos tecnológicos que es posible advertir en el desarrollo de la segunda etapa de "e-Japan Stratetegy", en realidad el aspecto más relevante es resultado de la alfabetización digital: la transformación de cibernautas ordinarios en prosumidores.

\section{El prosumidor. El actor comunicativo de la sociedad de la ubicuidad}

En la breve y fecunda historia de Internet, las remediaciones han sido frecuentes. Entre las remediaciones más significativas se destacan la introducción de la web en los primeros años de la década de los noventa, y el desarrollo de los blogs a finales de la misma década. La web, generosa aportación de Tim Berners-Lee -quien

transacciones en línea, tarjeta de crédito, identificación personal, dispositivo de acceso aeroportuario, etc. El aumento en el número de usuarios de Internet dependerá ahora de los dispositivos móviles y no del incremento que registre el número de computadoras disponibles con acceso a Internet. 
hoy se desempeña como director del World Wide Web Consortium-, aceleró la socialización de Internet. Los blogs o bitácoras permitieron que usuarios no expertos en informática fueran capaces de poder publicar contenidos en Internet.

El desarrollo de la web 2.0 admite ser considerado como un auténtico parteaguas histórico en la evolución de Internet. El desarrollo de la web 2.0 impuso importantes cambios tanto en el comportamiento como en los hábitos de consumo cultural de los cibernautas. El cibernauta accedió a la condición de prosumidor.

La palabra prosumidor -en inglés, prosumer-, es un acrónimo que procede de la fusión de dos palabras: "producer" (productor) y "consumer" (consumidor). El concepto "prosumidor" fue anticipado por Marshall McLuhan y Barrington Nevitt, quienes en el libro Take Today (1972), afirmaron que la tecnología electrónica permitiría al consumidor asumir simultáneamente los roles de productor y consumidor de contenidos.

En 1980 el destacado futurólogo Alvin Toffler introdujo formalmente el término prosumidor en el libro La tercera ola. El capítulo XX del referido libro, precisamente consigna el siguiente título: "El resurgimiento del prosumidor". Las actividades de los prosumidores -anticipó Toffler (1981, pp. 262-263)-, definirían el rumbo de la "economía invisible":

Durante la primera ola, la mayoría de las personas consumían lo que ellas mismas producían. No eran ni productores ni consumidores en el sentido habitual. Eran, en su lugar, lo que podría denominarse prosumidores. Fue la revolución industrial lo que, al introducir una cuña en la sociedad, separó estas dos funciones y dio nacimiento a lo que ahora llamamos productores y consumidores (...) si examinamos atentamente la cuestión, descubrimos los comienzos de un cambio fundamental en la relación mutua existente entre estos dos sectores o formas de producción. Vemos un progresivo difuminarse de la línea que separa al productor del consumidor. Vemos la creciente importancia del prosumidor. Y, más allá de eso, vemos aproximarse un impresionante cambio que transformará incluso la función del mercado mismo en nuestras vidas y en el sistema mundial.

En La tercera ola, Toffler afirma que el medio de comunicación más poderoso y masificador ha sido la televisión. El advenimiento de los prosumidores, sin embargo, anticiparía el fin de la era de los medios masificadores (Toffler, 1981, p. 167): "están desapareciendo los días de la omnipotente red centralizada que controla la producción de imágenes (...) los medios de comunicación de la tercera ola están destruyendo en un amplio frente el dominio ejercido por los dueños de los medios de comunicación de la segunda ola".

Efectivamente, para comprender el impacto de YouTube en la ecología cultural de la industria de la televisión, resulta indispensable reparar en el comportamiento de los prosumidores, tal como destacan Alvin y Heidi Toffler en el libro La revolución de la riqueza (2006, p. 99):

En palabras de Betsy Frank, vicepresidenta ejecutiva de investigación y planificación de MTV Networks, "se trata de un público que desea hacer su propia programación". Los nuevos instrumentos del consumidor ya otorgan a los teleespectadores el poder de cortar y pegar partes de programas para adecuarlos a sus preferencias

El desarrollo de la web 2.0 impuso importantes cambios tanto en el comportamiento como en los hábitos de consumo cultural de los cibernautas. El cibernauta accedió a la condición de prosumidor. 


\section{La web 2.0 ha propiciado la transformación de los consumidores pasivos en activos prosumidores que han impulsado importantes transformaciones en el mercado al apostar por el recurso de la conversación, comprendiendo a Internet como efectivo multiplicador del capital intelectual}

personales. Este desplazamiento continuo de los tiempos estándar de los huecos en la programación se acelerará a medida que las audiencias de los medios de comunicación, provistas de nuevas tecnologías, produzcan sus propios contenidos. Al mismo tiempo que los espectadores están creando sus propios contenidos, también exigen acceder a programas "a petición" antes que al horario establecido por los medios de comunicación. En palabras de William Randolph Hearst III, "la televisión basada en el proveedor está muerta".

En el año 2000, Rick Levine, Christopher Locke, Doc Searls y David Weinberger, autores del libro The cluetrain manifesto. The end of business as usual, destacaron cómo la web 2.0 ha propiciado la transformación de los consumidores pasivos en activos prosumidores que han impulsado importantes transformaciones en el mercado al apostar por el recurso de la conversación, comprendiendo a Internet como efectivo multiplicador del capital intelectual ${ }^{7}$.

En el libro Capital Digital. El poder de las redes de negocios, Don Tapscott, David Ticoll y Alex Lowy (2001), emplearon la palabra prosumption (prosumo) para definir el comportamiento de per-

7 Resulta elocuente la primera de las 95 tesis consignadas en el libro The cluetrain manifesto. The end of business as usual: los mercados son conversaciones. sonas cuyos conocimientos y trabajo aportan valor a las redes de negocios (b-web) en Internet.

Los prosumidores han empezado a asumir roles de liderazgo en la llamada sociedad-red. Las multitudes inteligentes (smart mobs) que refiere Howard Rheingold son posibles gracias a la formidable capacidad de convocatoria que han alcanzado algunos prosumidores.

\section{Conclusión. La contribución de los prosumers en la remediación de Internet}

"Societies have always been shaped more by the nature of the media by wich men communicate than the content of the communication" (McLuhan, 1967, p. 8).

De acuerdo con Noam Chomsky (1997, p. 60), los medios masivos convencionales promueven la pasividad dirigida sobre sus audiencias:

Como mencioné antes, no desean gente que tome decisiones o participe; quieren una población de consumidores y espectadores políticos pasivos y obedientes; una comunidad tan atomizada y aislada que le resulte imposible reunir sus limitados recursos para convertirse en una fuerza independiente, poderosa que denuncie la concentración de poder.

Si el usuario de los medios de comunicación convencionales se ve obligado a soportar la "pasividad dirigida" que denuncia Chomsky, la ecología cultural en la cual se desenvuelve el prosumidor estimula su autonomía. Atento a tal fenómeno, Thomas Friedman, autor del libro La tierra es plana. Breve historia del mundo globalizado del siglo XXI (2005, p. 164) afirma: “Jamás en la historia del planeta tanta gente ha tenido la posibilidad de buscar por si misma tanta información acerca de tantos temas o acerca de tanta gente". 
Entre las variables que permiten establecer diferencias significativas entre el comportamiento de los prosumidores con respecto al comportamiento que observan simples usuarios de Internet, se destacan el in-forming y la colaboración. Si la búsqueda de la verdad efectivamente nos hará libres, los prosumidores, a diferencia del usuario de los medios convencionales -cuyo acceso a la información en buena medida suele ser dosificado por las instituciones históricas y por el sistema convencional de medios de comunicación-, acostumbran involucrarse en la búsqueda de respuestas.

La capacidad de búsqueda del prosumidor representa una evidente afirmación de su independencia, tal como afirma Erich Schmidt, director general de Google, citado por Friedman (2005, p. 169):

La búsqueda es una tarea tan personal que revierte en una emancipación sin igual del ser humano (...) es lo contrario de que te digan o te enseñen. Se trata de dotarse de medios que te emancipen, es la atribución de poder al individuo para que haga lo que considere mejor con la información que desee.

Entre las interesantísimas tesis contenidas en el libro Futuro presente. El futuro es atreverse hoy. 101 Ideas-Fuerza para entender las próximas décadas, Alfons Cornella y Sergi Rucabado destacan el "x-casting”. El $x$-casting designa un fenómeno recurrente en el imaginario de la sociedad de la ubicuidad: toda persona -en cualquier momento y en cualquier lugar- puede introducir información a Internet. Toda persona que pueda acceder a Internet representa un potencial prosumidor. Un hecho tan simple representa una profunda revolución en la economía política de los sistemas de comunicaciones. El in-forming solo así es posible. De acuerdo con Friedman (2005, p. 198), el in-forming: es la capacidad de crear y desplegar tu propia cadena de suministro, una cadena de suministro de información, de conocimientos y de entretenimiento. El in-forming tendría que ver con una colaboración individual: tú mismo eres el que investiga, edita o elige el entretenimiento, siguiendo tus propias pautas y valiéndote de tu propia capacidad y medios, sin necesidad de acudir a la biblioteca o al cine o a una cadena de televisión. El in-forming es búsqueda de conocimiento.

La colaboración resulta medular en el comportamiento de los prosumidores. Los nuevos ambientes comunicativos que desplaza consigo el desarrollo de la web 2.0 -la blogósfera, por ejemplo-, se proyectan como escenarios propicios para el activismo de redes de prosumidores, las cuales han denunciado, por ejemplo, prácticas inescrupulosas de algunas marcas cuyo comportamiento no precisamente corresponde a lo dispuesto en sus códigos de ética. En el prólogo de El libro negro de las marcas, por ejemplo, Klaus Werner y Hans Weiss refieren que una gran cantidad de la información consignada en el libro les fue proporcionada por redes organizadas de prosumidores a través de Internet.

Entre las principales cadenas de socialización del conocimiento que ha propiciado el desarrollo de Internet se destaca Google, la marca emblemática de la economía del conocimiento. De acuerdo con Friedman (2005, p. 167), los fundadores de Google -Larry Page y Sergen Brin-, crearon la empresa en septiembre de 1998, considerando los siguientes propósitos:

El $x$-casting designa un fenómeno recurrente en el imaginario de la sociedad de la ubicuidad: toda persona -en cualquier momento y en cualquier lugar- puede introducir información a Internet. 
Los fundadores de Google vieron que a finales de los 90 aparecían en internet cientos de miles de páginas web nuevas cada día, y que los motores de búsqueda existentes, que tendrían que buscar palabras clave, no podían seguir ese ritmo de crecimiento. Brin y Page, que se conocieron en 1995 cuando estudiaban informática en la Universidad de Stanford, desarrollaron una fórmula matemática que clasificaba una página web según la cantidad de páginas web vinculadas a ella, partiendo de la hipótesis de que cuantas más personas creasen un vínculo con determinada página, más importante debería ser ésta.

De acuerdo con lo asentado en la página corporativa de Google ${ }^{8}$, la cual fue consultada el 31 de marzo de 2008: "el objetivo de Google consiste en organizar información proveniente de todo el mundo y hacerla accesible y útil de forma universal". Google es resultado de la colaboración. La gente asume que la información que está buscando -destaca Friedman (2005, p. 166) - se encuentra en Google o en Internet: "y que todo se reduce a que los expertos en tecnología vayan simplificando la manera de acceder a ella, cada vez con menos pasos (...) La democratización de la información está teniendo un impacto profundo en la sociedad".

Debemos reconocer a los prosumidores como los actores comunicativos de la sociedad de la ubicuidad. El papel de los prosumidores resultará definitivo en las siguientes remediaciones

"El objetivo de Google consiste en organizar información proveniente de todo el mundo y hacerla accesible y útil de forma universal". Google es resultado de la colaboración.

8 http://www.google.com/intl/es/corporate/index.html que experimentará Internet, medio que definitivamente admite ser comprendido como lógica extensión de la inteligencia humana.

\section{Referencias}

Chomsky, N. (1997). Secretos, mentiras y democracia. México: Siglo Veintiuno Editores.

Cornella, A. y Rucabado, S. (2006). El futuro es atreverse hoy. 101 Ideas-Fuerza para entender las próximas décadas. España: Ediciones Deusto.

Friedman, T. (2005). La Tierra es plana. Breve historia del mundo globalizado del siglo XXI. España: mr Ediciones.

Levinson, P. (1997). The soft age. A natural history ad future of the information revolution. London: Routledge.

Levinson, P. (1999). Digital McLuhan. A guide to the information millennium. New York: Routledge.

Levinson, P. (2004). Cellphone. The story of the world's most mobile medium and how it has transformed everything. USA: Palgrave MacMillan.

McLuhan, M. (1996). Comprender los medios de comunicación. Las extensiones del ser humano. Barcelona: Paidós Comunicación.

McLuhan, M. y Nevitt, B. (1972). Take Today: the Executive As Dropout. New York: Harcourt Brace Jovanovish.

McLuhan, M. (1996). Comprender los medios de comunicación. Las extensiones del ser humano. Barcelona: Paidós Comunicación.

McLuhan M. y Fiore, Q. (1967). The medium is the massage. An inventory of effects. New York: Bantham Books. 
Tapscott, D., Tocll, D. y Lowy, A. (2001). Capital digital. El poder de las redes de negocios. España: Taurus Digital.

Toffler, A. (1981). La tercera ola. México: Edivisión.
Toffler, A. y Toffler, H. (2006). La revolución de la riqueza. España: Deusto.

Werner, K. y Weiss, H. (2006). El libro negro de las marcas. España: DeBOLSILLO. 